\title{
Central Diabetes Insipidus in an Extremely-Low-Birth- Weight Preterm Infant with Suspected Ectopic Posterior Lobe of the Pituitary Gland
}

Yung Zu Park, $\mathrm{MD}^{1}$, Inseong Hwang, $\mathrm{MD}^{1}$, Sung-Ha Kim, $\mathrm{MD}^{1}$, Sook Min Hwang, $\mathrm{MD}^{2}$, Tae-Jung Sung, $\mathrm{MD}, \mathrm{PhD}^{1}$, and Hye Jin Lee, $\mathrm{MD}^{1}$

Departments of ${ }^{1}$ Pediatrics and ${ }^{2}$ Radiology, Hallym University Kangnam Sacred Heart Hospital, Hallym University College of Medicine, Seoul, Korea

\section{ABSTRACT}

Central diabetes insipidus (CDI) is extremely rare in neonates, especially in extremelylow-birth-weight infants, and most cases are secondary to conditions, such as ischemic or hemorrhagic brain damage. Here, we report a case of CDI in a 530-g infant born at $23^{+3}$ weeks of gestation, with suspected ectopic posterior pituitary gland. Hypernatremia was noticed at $33^{+6}$ weeks of postmenstrual age, and it persisted with increased volumes of diluted urine, despite adequate sodium intake. Serum and urine osmolality returned to the normal range after administration of a desmopressin injection. The bright spot of the posterior pituitary was absent, and brain magnetic resonance imaging suggested an ectopic posterior pituitary gland. At the time of writing this manuscript, the patient was on oral desmopressin medication without complications at the corrected age of 8 months. Through this report, we emphasize that although CDI is extremely rare in premature infants, it should be suspected when hypernatremia and polyuria that are unexplained by other causes are noted.

Key Words: Diabetes insipidus, neurogenic; Pituitary gland, posterior; Extremely low birth weight; Infant, newborn; Hypernatremia

\section{INTRODUCTION}

Central diabetes insipidus (CDI) is a disease due to the deficiency of arginine vasopressin (AVP), which is secreted through the hypothalamic neurohypophysis (posterior pituitary). CDI results from various conditions that affect the hypothalamic-neurohypophysial system ${ }^{1)}$. Deficiency of AVP causes excessively dilute urine, which can be lethal if the patients cannot control their own water intake.

$\mathrm{CDI}$ is extremely rare in neonates ${ }^{2}$. Transient hypernatremia with dehydration can occur during the neonatal period, especially in extremely-low-birth-weight (ELBW) and preterm
Received: 11 September 2019

Revised: 18 October 2019

Accepted: 18 October 2019

Correspondence to: Hye Jin Lee, MD

Department of Pediatrics, Hallym University Kangnam Sacred Heart Hospital, Hallym University College of Me dicine, 1 Singil-ro, Yeongdeungpo-gu, Seoul 07441, Korea

Tel: +82-2-6960-1300

Fax: +82-2-849-4469

E-mail: hjleeped@hallym.or.kr

Copyright(c)

By Korean Society of Neonatology.

All right reserved.

This is an Open-Access article distributed under the terms of the Creative Commons At tribution Non-Commercial License (http:// creativecommons.org/licenses/by-nc/4.0), which permits unrestricted non-commercial use, distribution, and reproduction in any medium, provided the original work is pro perly cited. 
newborns, and it is associated with unnoticeable water loss, high urine output, and a reduced ability to excrete sodium ${ }^{3)}$. Hence, it is difficult to diagnose CDI in neonates, and this could result in delayed treatment. Here, we report a case of CDI diagnosed in a preterm ELBW infant.

\section{CASE REPORT}

A preterm female infant was delivered at a gestational age of 23 weeks and 3 days owing to fetal distress associated with preterm premature rupture of the membranes (PPROM). Her parents were not consanguineous and did not have any significant medical history. The mother was a 32-year-old primipara who had had a normal antenatal examination. The infant was vaginally delivered, and the Apgar scores were 1 at 1 minute, 2 at 5 minutes, and 4 at 10 minutes. Her birth weight was $530 \mathrm{~g}$ (35th percentile), length was $30.3 \mathrm{~cm}$ (45th percentile), and head circumference was $20.1 \mathrm{~cm}$ (25th percentile). She had low-set ears but no other definite dysmorphic features. She did not have cleft lip, cleft palate, or a high arched palate.

The infant was immediately intubated with surfactant and admitted to our newborn intensive care unit. She was supported with a mechanical ventilator for 57 days, followed by non-invasive positive pressure ventilation for a further 98 days. She was also treated for retinopathy of prematurity, bronchopulmonary dys plasia (BPD), secondary pulmonary hypertension, and postnatal cytomegalovirus (CMV) infection. She had patent ductus arterio sus and minimal intraventricular hemorrhage.

Significant hypernatremia was first noticed on day 74 of the infant's life at $33^{+6}$ weeks postmenstrual age. Serum sodium level was $156 \mathrm{mEq} / \mathrm{L}$ (normal range, 135 to 145), and the diuresis rate was $4.1 \mathrm{~mL} / \mathrm{kg} / \mathrm{hr}\left(1,149.1 \mathrm{~mL} / \mathrm{m}^{2} /\right.$ day $)$ without glycosuria. De spite no sodium input, except for oral preterm formula intake up to $145 \mathrm{~mL} / \mathrm{kg} /$ day (sodium $2.56 \mathrm{mEq} / \mathrm{kg}$ /day), the hypernatremia persisted. As the hypernatremia was accompanied by elevated C-reactive peptide ( $10.7 \mathrm{mg} / \mathrm{L}$ [normal range, 0.0 to 3.0]), an intravenous (IV) line was connected for antibiotic administration for any suspected infection. No sodium was supplied through the IV solution; the oral intake was reduced as the IV fluid volume increased, resulting in a net decreased sodium supply (1.33 $\mathrm{mEq} / \mathrm{kg} /$ day). Laboratory examinations on day 77 of life revealed persistent hypernatremia (155 mEq/L), low urine sodium level (21 mEq/L [normal range, >40]), increased serum osmolality (315 $\mathrm{mOsm} / \mathrm{kg}$ [normal range, 276 to 292]), low urine osmolality (97 $\mathrm{mOsm} / \mathrm{kg}$ [normal range, 40 to 1,400$]$ ), and low urine specific gravity (1.005 [normal range, 1.002 to 1.030 ]) compared to serum sodium and osmolality. Other serum electrolyte and chemistry levels including that of glucose were in the normal range. A therapeutic trial with subcutaneous desmopressin $(0.5 \mu \mathrm{g}, 0.34$ $\mu \mathrm{g} / \mathrm{kg}$ ) was performed, and laboratory examination performed 2 hours after desmopressin injection showed a serum sodium level of $152 \mathrm{mEq} / \mathrm{L}$, plasma osmolality of $315 \mathrm{mOsm} / \mathrm{kg}$, and urine osmolality of $436 \mathrm{mOsm} / \mathrm{kg}$. The infant was administered oral desmopressin ( $0.05 \mathrm{mg}, 0.013 \mathrm{mg} / \mathrm{kg} /$ dose $)$ every 12 hours; subsequently, her electrolyte level, serum osmolality, and urine osmolality normalized (Figure 1).

Considering the possibility of transient diabetes insipidus, administration of oral desmopressin was discontinued on day 155 of the infant's life. After one dose of desmopressin was by passed, polyuria of $6.7 \mathrm{~mL} / \mathrm{kg} / \mathrm{hr}\left(2,293.3 \mathrm{~mL} / \mathrm{m}^{2} /\right.$ day $)$ occurred; therefore, desmopressin was restarted (Figure 1) and its dose was adjusted, with close monitoring of urine output and serum sodium levels thereafter.

Thyroid function tests conducted on day 28 day of life showed low free thyroxine (T4) levels of $0.52 \mathrm{ng} / \mathrm{dL}$ (normal range, 0.89 to

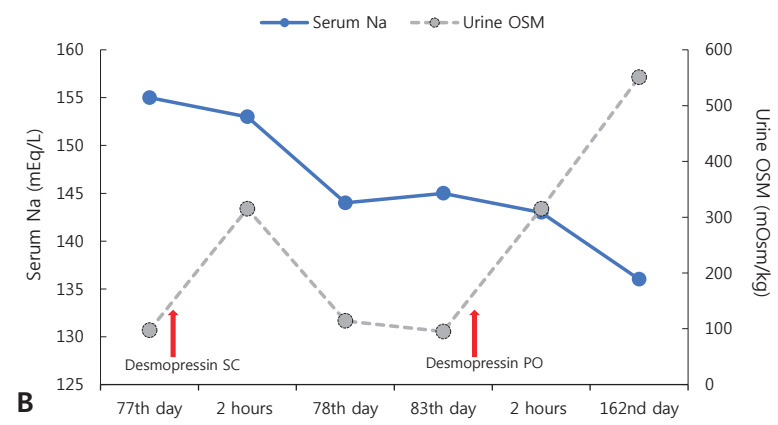

Figure 1. (A) Effect of desmopressin (arrows) on urine output ( $\mathrm{mL} / \mathrm{kg} / \mathrm{hr})$, (B) serum sodium concentration (mEq/L), and urine osmolality (OSM) (mOsm/kg). Abbreviation: SC, subcutaneous; PO, per os. 
1.76), low total triiodothyronine (T3) levels of $54 \mathrm{ng} / \mathrm{dL}$ (normal range, 60 to 181), and elevated thyroid stimulating hormone (TSH) levels of $>150 \mathrm{IU} / \mathrm{mL}$ (normal range, 0.550 to 4.780 ). The infant was started on levothyroxine $(15 \mu \mathrm{g} / \mathrm{kg} / \mathrm{dose})$ treatment. On day 162 of life, adrenocorticotropic hormone and morning cortisol levels of the infant were $15.0 \mathrm{pg} / \mathrm{mL}$ (normal range, 7.2 to 63.3 ) and $6.17 \mu \mathrm{g} / \mathrm{dL}$ (normal range, >6.34), respectively. One month later, when she was re-admitted for viral infection, her cortisol level was $22 \mu \mathrm{g} / \mathrm{dL}$. The serum insulin-like growth factor 1 level was not measured.

Transfontanellar sonography examinations were performed regularly from day 4 of life until discharge, and they continuously showed grade I intracranial hemorrhage, without any major deterioration. Sonography showed absence of normal echogenic cavum septum pellucidum, which was later confirmed using brain magnetic resonance imaging (MRI) performed at the age of 5 months (Figure 2A). MRI revealed absence of the normal posterior pituitary bright spot and a tiny high-signal intensity at the pituitary stalk on the sagittal T1-weighted image, which indicated possible ectopic location of the posterior lobe of the pituitary gland (Figure 2B). The optic nerve was present (Figure 2C).

Serological tests for syphilis, toxoplasmosis, CMV, and rubella were performed at admission owing to PPROM, and all results were negative. CMV urine polymerase chain reaction (PCR) and culture surveillance tests were performed once every 2 weeks. Active CMV infection was diagnosed and treated based on elevated liver function test results on day 86 of life, after starting treatment for CDI. Blood cultures provided no evidence for bacterial infection during hospitalization.
The infant was discharged at the age of 6 months ( 2 months of corrected age), with a weight of 3,190 g (3rd percentile), height of $53 \mathrm{~cm}$ (3rd percentile), and head circumference of $35 \mathrm{~cm}$ (3rd percentile); she was prescribed $0.02 \mathrm{mg}$ of oral desmopressin $(0.006 \mathrm{mg} / \mathrm{kg} / \mathrm{dose})$ every 12 hours. Her parents were instructed to monitor her daily oral intake, urine output, and body weight. In addition, they were warned against administration of desmopressin to the infant before excretion of diluted urine. The last urine volume, urine osmolality, and serum sodium tests performed at the outpatient clinic showed normal results at the age of 8 months.

\section{DISCUSSION}

$\mathrm{CDI}$ is a disease characterized by excretion of large volumes of dilute urine owing to deficiency of AVP. AVP is synthesized in the supraoptic and paraventricular nuclei of the hypothalamus and transported to the posterior pituitary to be secreted into the blood stream. After being released into the circulation, AVP binds to a target receptor in the kidney and facilitates water reabsorption. It is normally regulated by plasma osmolality; however, patients with CDI show $80 \%$ to $90 \%$ decreased secretion of AVP, and the kidney is no longer capable of concentrating urine, resulting in low urine osmolality and polyuria ${ }^{1,2}$.

The known causes of CDI include genetic factors, trauma resulting from surgery or an accident, holoprosencephaly, septooptic dysplasia (SOD), craniopharyngioma, germinoma, Langerhans cell histiocytosis, autoimmune disease, and infection ${ }^{1)}$. In most cases, CDI in neonates has been caused by a complication
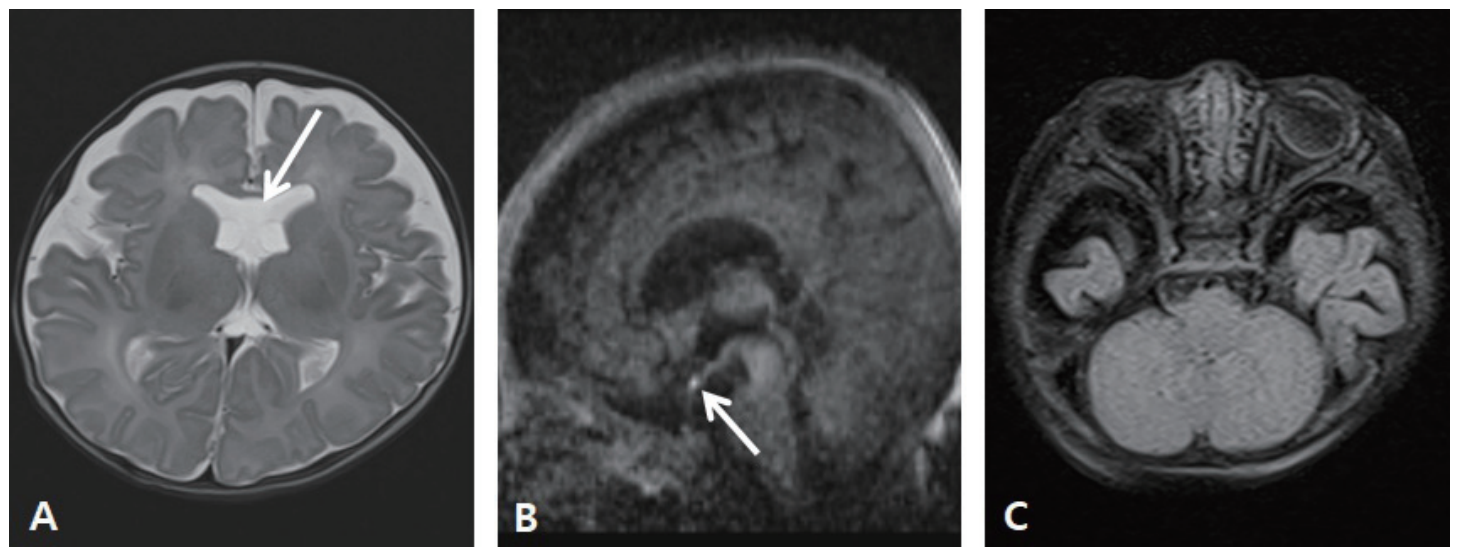

Figure 2. Magnetic resonance imaging (MRI) findings. (A) Axial T2-flair MRI of the brain shows absent cavum septum pellucidum (arrow). (B) Sagittal T1-weighted image shows the pituitary stalk, absent posterior pituitary bright spot, and hyperintensity lesion (arrow), suggesting the possibility of ectopic posterior pituitary lobe. (C) Axial T2-flair image demonstrates both optic nerves. 
of intrauterine and perinatal disease ${ }^{3)}$. Idiopathic CDI is thought to account for $12 \%$ to $24 \%$ of cases $^{2)}$. Previous reports of CDI in preterm infants ${ }^{2-9)}$, excluding traumatic or ischemic brain injuries, are summarized in Table 1.

Clinical manifestations such as polyuria, polydipsia, and irritability may provide important clues for the diagnosis of CDI. However, such symptoms may not be obvious in preterm infants. Persistent hypernatremia was the only initial sign in the present case. Although fluid and electrolyte disorders are frequent in preterm infants, we did not ignore the laboratory test results and conducted further studies. A water deprivation test is not recom mended in neonates ${ }^{10)}$. As a therapeutic trial, we subcutaneously administered desmopressin ( 0.5 and $0.34 \mu \mathrm{g} / \mathrm{kg}$ ) to distinguish between nephrogenic and CDI. Serum and urine osmolality resolved to the normal range after desmopressin administration, and a diagnosis of CDI was confirmed.

The infant underwent a diagnostic work-up for the etiology of CDI, including brain imaging studies, signs of infection, and medication review. The posterior pituitary bright spot was ab- sent, and MRI suggested ectopic posterior lobe of the pituitary gland. Typically, the posterior pituitary shows a hyperintense signal on T1-weighted MRI. Although MRI finding of an absent posterior pituitary hyperintense signal is not diagnostic, it is a well-known characteristic of $\mathrm{CDI}^{11-13)}$. Ectopic posterior pituitary was observed in three neonates in a study reported by Djermane et al. ${ }^{8)}$. An ectopic posterior pituitary may be the cause of CDI due to a structural anomaly in the current patient. Patients with isolated ectopic posterior pituitary without other midline defects, such as SOD or absent corpus callosum, feel excessively thirsty, eventually leading to polydipsia in older children. However, neonates, who cannot access water by themselves, are at a risk of dehydration and hypernatremia owing to delayed detection of $\mathrm{CDI}^{13)}$.

Although the cavum septum pellucidum may be absent occur in preterm neonates without clinical significance, it requires further evaluation with respect to other brain anomalies ${ }^{14)}$. In cases of absent cavum septum pellucidum with CDI, SOD should be differentially diagnosed. SOD can be diagnosed when the patient

Table 1. Case Reports of Central Diabetes Insipidus in Preterm Infants

\begin{tabular}{|c|c|c|c|c|c|c|c|c|c|}
\hline Variable & Case 1 & Case 2 & Case 3 & Case 4 & Case 5 & Case 6 & Case 7 & Case 8 & Our case \\
\hline Reference no. & 2) & 3) & 4) & 5) & 6) & 7) & 8) & 9) & \\
\hline Country & France & Brazil & Turkey & France & USA & Singapore & France & Korea & Korea \\
\hline Sex & Male & Male & Female & Unknown & Male & Male & Male & Male & Female \\
\hline Gestational age (wk) & 26 & 31 & 28 & 30 & 23 & 25 & 30 & 25 & 23 \\
\hline Age of first symptoms (day of life) & 17 & 3 & 11 & 10 & 21 & 69 & 10 & 71 & 74 \\
\hline Maximum diuresis (mL/kg/hr) & 10 & 7.4 & 6.5 & 7.3 & 9 & Unknown & Unknown & 5.6 & 6.4 \\
\hline Highest level of natremia (mEq/dL) & 173 & 146 & 170 & 155 & 164 & 157 & 157 & 160 & 157 \\
\hline Urine osmolality (mOsm/kg) & 104 & 94 & 165 & 115 & 178 & 181 & 125 & 117 & 97 \\
\hline Anterior pituitary hormone deficiency & Normal & Normal & Normal & Normal & Unknown & Normal & Normal & Partial GH & Normal \\
\hline Transfontanelle sonography & IVH GI & IVH GIII & Unknown & IVH GII & IVH GI GII & IVH GIII & Unknown & Normal & IVH GI \\
\hline Brain MRI findings & $\begin{array}{c}\text { Absent } \\
\text { PPHS }\end{array}$ & $\begin{array}{c}\text { Absent } \\
\text { PPHS }\end{array}$ & Normal & Normal & Normal & Unknown & $\begin{array}{c}\text { Absent } \\
\text { PPHS }\end{array}$ & $\begin{array}{c}\text { Absent } \\
\text { PPHS }\end{array}$ & $\begin{array}{c}\text { Absent } \\
\text { PPHS, } \\
\text { ectopic PP }\end{array}$ \\
\hline Preceding events & No & No & No & No & No & No & & $\begin{array}{c}\text { CMV } \\
\text { infection }\end{array}$ & No \\
\hline
\end{tabular}


has two or more of the following: optic nerve hypoplasia, pituitary hormone abnormalities, or midline brain defects ${ }^{15)}$. In the present case, although the presence of an optic nerve was demonstrated on MRI and anterior pituitary hormone secretion was normal, follow-up MRI and anterior hormone monitoring were necessary. Serial transfontanellar sonography demonstrated Grade 1 intraventricular hemorrhage, which is common in preterm births. The intraventricular hemorrhage did not progress and was minimal at discharge.

The anterior pituitary function of the infant was intact at the time of evaluation. She was started on levothyroxine medication for primary hypothyroidism; her TSH was elevated, with low free T4 and total T3 levels ${ }^{16)}$. The morning cortisol level, measured on day 163 of life, was slightly low. However, there were no symp. toms of adrenal insufficiency, and considering that a circadian rhythm in cortisol secretion requires approximately 6 months to stabilize $^{10)}$, the cortisol levels were followed up without medication. Serum cortisol level measured in a stress situation 1 month later was normal. The growth of such infants should be monitored, and the secretion of growth hormone should be evaluated. The levels of anterior pituitary hormones, which are considered to be normal in patients with CDI at the time of diagnosis, should also be monitored regularly.

As a case of CDI after CMV infection has previously been reported, this association was also considered in the present case ${ }^{9)}$. Although CMV urine PCR showed positive results before the diagnosis of CDI, it is difficult to conclude that CMV infection was the cause of CDI, as the symptoms of infection appeared later and were mild.

As the patient was an ELBW premature infant, she had a complicated hospital course, and a variety of medication was used for treatment. Diuretics were intermittently used for treatment of acute kidney injury; however, the last use of diuretics was 50 days before the diagnosis of CDI. Dopamine was injected to control hypotension early in hospitalization; however, it was not associated with polyuria. Corticosteroids were also used thrice during the treatment. A total of $10.6 \mathrm{mg} / \mathrm{kg}$ of hydrocortisone was used as maintenance treatment for shock that occurred after both incidents of pneumothorax from day 12 to day 25 of life. From day 33 to day 43 of life, $5.5 \mathrm{mg} / \mathrm{kg}$ of dexamethasone was used to control BPD and assist extubation. Prednisolone $(1 \mathrm{mg} / \mathrm{kg}$ ) was used once or twice a day for treatment of BPD from day 163 to day 172 of life. Exogenous glucocorticoids can cause a physiological decrease in the secretion of AVP, which has been reported to cause CDI in some cases; however, the symptoms disappeared with discontinuation of glucocorticoid administration ${ }^{17,18)}$. In the present case, signs of CDI persisted even after discontinuation of glucocorticoid administration.

In conclusion, we report a case of an ELBW preterm infant with CDI who was diagnosed early and successfully treated with desmopressin. Although CDI is a rare cause of hypernatremia in the neonatal period, especially in preterm infants, it should be considered when hypernatremia is not corrected and if diluted urine persists despite proper fluid therapy.

\section{ARTICLE INFORMATION}

\section{Ethical statement}

The study protocol was approved by the Institutional Review Board of Hallym University Kangnam Sacred Heart Hospital (Approval number: 2019-09-002). As this was a retrospective case report, the requirement for written informed consent was waived.

\section{Conflicts of interest}

No potential conflict of interest relevant to this article was reported.

\section{Author contributions}

Conception or design: Y.Z.P., S.H.K., T.J.S., H.J.L.

Acquisition, analysis, or interpretation of data: Y.Z.P., I.H., S.H.K., S.M.H., H.J.L.

Drafting the work or revising: Y.Z.P., S.M.H., T.J.S., H.J.L.

Final approval of the manuscript: Y.Z.P., I.H., S.H.K., S.M.H., T.J.S., H.J.L.

\section{ORCID}

Yung Zu Park https://orcid.org/0000-0003-2370-6746

Hye Jin Lee https://orcid.org/0000-0002-7925-5238

\section{Acknowledgments}

None

\section{REFERENCES}

1. Di Iorgi N, Napoli F, Allegri AE, Olivieri I, Bertelli E, Gallizia A, 
et al. Diabetes insipidus: diagnosis and management. Horm Res Paediatr 2012;77:69-84.

2. Biset A, Claris O. Idiopathic central diabetes insipidus in an extreme premature infant: a case report. Arch Pediatr 2018;25: 480-4.

3. Ferlin ML, Sales DS, Celini FP, Martinelli Junior CE. Central diabetes insipidus: alert for dehydration in very low birth weight infants during the neonatal period. A case report. Sao Paulo Med J 2015;133:60-3.

4. Hanta D, Torer B, Temiz F, Kilicdag H, Gokce M, Erdogan O. Idiopathic central diabetes insipidus presenting in a very low birth weight infant successfully managed with lyophilized sublingual desmopressin. Turk J Pediatr 2015;57:90-3.

5. Quetin F, Garnier H, Brauner R, Vodovar M, Magny JF. Persistent central diabetes insipidus in a very low birth weight infant. Arch Pediatr 2007;14:1321-3.

6. Stapleton G, DiGeronimo RJ. Persistent central diabetes insipidus presenting in a very low birth weight infant successfully managed with intranasal dDAVP. J Perinatol 2000;20:132-4.

7. Sng A, Loke KY, Lim Y. A case of neonatal central diabetes insipidus in a premature infant: challenges in diagnosis and management. Case Rep J 2018;2:010.

8. Djermane A, Elmaleh M, Simon D, Poidvin A, Carel JC, Leger J. Central diabetes insipidus in infancy with or without hypothalamic adipsic hypernatremia syndrome: early identification and outcome. J Clin Endocrinol Metab 2016;101:635-43.

9. Hong YR. Central diabetes insipidus associated with symptomatic cytomegalovirus infection in an extremely low birth weight infant. J Korean Soc Neonatol 2012;19:158-62.

10. Kurtoglu S, Ozdemir A, Hatipoglu N. Neonatal hypopituitarism: approaches to diagnosis and treatment. J Clin Res Pediatr Endocrinol 2019;11:4-12.

11. Maghnie M, Villa A, Arico M, Larizza D, Pezzotta S, Beluffi G, et al. Correlation between magnetic resonance imaging of posterior pituitary and neurohypophyseal function in children with diabetes insipidus. J Clin Endocrinol Metab 1992;74:795800.

12. Shin JH, Lee HK, Choi CG, Suh DC, Kim CJ, Hong SK, et al. MR imaging of central diabetes insipidus: a pictorial essay. Korean J Radiol 2001;2:222-30.

13. Ozata M, Tayfun C, Kurtaran K, Yetkin I, Beyhan Z, Corakci A, et al. Magnetic resonance imaging of posterior pituitary for evaluation of the neurohypophyseal function in idiopathic and autosomal dominant neurohypophyseal diabetes insipidus. Eur Radiol 1997;7:1098-102.

14. Sundarakumar DK, Farley SA, Smith CM, Maravilla KR, Dighe MK, Nixon JN. Absent cavum septum pellucidum: a review with emphasis on associated commissural abnormalities. Pediatr Radiol 2015;45:950-64.

15. Webb EA, Dattani MT. Septo-optic dysplasia. Eur J Hum Genet 2010;18:393-7.

16. Rastogi MV, LaFranchi SH. Congenital hypothyroidism. Orphanet J Rare Dis 2010;5:17.

17. Bahr V, Franzen N, Oelkers W, Pfeiffer AF, Diederich S. Effect of exogenous glucocorticoid on osmotically stimulated antidiuretic hormone secretion and on water reabsorption in man. Eur J Endocrinol 2006;155:845-8.

18. Ohta M, Kimura T, Ota $K$, Shoji M, Inoue $M$, Sato $K$, et al. Glucocorticoid-induced central diabetes insipidus in a case of malignant lymphoma. Tohoku J Exp Med 1991;163:245-54. 